Review

\title{
Delay in adjuvant radiation treatment and outcomes of breast cancer - a review
}

\author{
N. Hébert-Croteau ${ }^{1,2}$, C.R. Freeman ${ }^{3}$, J. Latreille ${ }^{2,4}$, and J. Brisson ${ }^{1,5}$ \\ ${ }^{1}$ Institut national de santé publique du Québec, Montréal; ${ }^{2}$ Centre de recherche, Hôpital Charles-Lemoyne, \\ Greenfield Park; ${ }^{3}$ Department of radiation oncology, McGill University Health Centre, Montréal; ${ }^{4}$ Centre intégré \\ de lutte contre le cancer de la Montérégie (CICM), Hôpital Charles-Lemoyne, Greenfield Park; ${ }^{5}$ Unité de recherche \\ en santé des populations et Centre des maladies du sein Deschênes-Fabia, Hôpital du Saint-Sacrement du Centre \\ hospitalier affilié (CHA) universitaire de Québec, Québec, Canada
}

Key words: breast neoplasms, combined modality therapy, radiotherapy, time factors, treatment delay

\section{Summary}

Recent meta-analyses have shown the importance of locoregional control as a long-term determinant of breast cancer survival. Whether factors related to the delivery of radiotherapy, such as delay, dose, fractionation or irradiated volume, are associated with outcome remains unclear. We performed a critical review of the literature on delay to radiation using a computerized search of papers published between 1985 and 2000. Periods of accrual, details of radiotherapy, surgical and systemic treatment, and information on prognostic factors were noted. Studies on sequencing of adjuvant therapy were compared to studies on delay to radiation, classified according to whether or not patients also received chemotherapy. Comparisons of patients receiving systemic therapy to individuals spared this option were considered uninformative since the impact of delaying radiation is then highly confounded by systemic treatment received. The single published experimental study on sequencing suggests that delay to radiation may compromise local control, and this is consistent with a few retrospective reports on delay to radiotherapy among patients receiving chemotherapy. However, indirect evidence from two randomized clinical trials of chemotherapy, and the majority of observational studies on delay to radiotherapy, suggest that it has no impact on either local, distant control or survival. Factors, methodological, and others, that could explain these inconsistencies are discussed. No study restricted to patients at low risk of recurrence suggested an impact of delaying radiation. Short chemotherapy regimens are likely to represent a safe option with respect to outcome of radiation treatment.

Abbreviations: RT: radiation therapy; CT: chemotherapy; HT: hormonal therapy; BCS: breast-conserving surgery; ER: estrogen receptors; OR: odds ratio; IBCSG: International Breast Cancer Study Group; vs: versus; NS: nonsignificant ( $p$-value $>0.05$ ); M: methotrexate; F, 5-FU: fluorouracil; C: cyclophosphamide; A: doxorubicin; E: epirubicin; P: prednisone; V: vincristine

\section{Introduction}

Radiation therapy (RT) following conservative surgery for breast cancer was established as the standard of care in the early 1990s [1, 2]. A recent expert panel also recommended postmastectomy radiotherapy in patients with four or more positive axillary lymph nodes, with T3 or stage III tumors [3]. Such treatment reduces the risk of local or locoregional re- lapse. However, different views have prevailed about the importance of local control as a determinant of long-term survival for this disease [4]. In addition to recent clinical trials among high-risk patients [5-7], two meta-analyses have provided new information on this relationship [8,9]. Whelan et al. [8] combined data from 18 trials where patients also received systemic adjuvant therapy and showed that radiotherapy reduced the risk of local recurrence, any recurrence, 
and death. In addition, both timing of radiotherapy and radiation technique were shown to influence outcomes of treatment. The odds ratio (OR) for mortality in trials in which radiation was administered within 6 months of starting chemotherapy was $0.78,95 \%$ confidence interval $(0.69,0.89)$, compared with $1.14,(0.80,1.62)$ in trials with delayed radiation. The Early Breast Cancer Trialists' Collaborative Group update at 20 years of follow-up of the meta-analysis of 40 unconfounded trials of radiotherapy for early breast cancer [9] also showed a significant disease-specific mortality reduction (20-year survival: $53.4 \%$ radiotherapy versus $48.6 \%$ controls, $p=0.0001)$, in addition to a reduction of about two thirds in local recurrences $(10.4 \%$ radiotherapy versus $30.1 \%$ controls, $p<0.00001$ ) associated with radiotherapy. Overall 20-year mortality was similar in women receiving or not radiation as a result of an increase in the number of deaths unrelated to breast cancer in irradiated women, especially cardiovascular mortality. The proportional effect of radiation therapy on breast cancer mortality was independent of age, stage, and nodal status, as well as treatment modalities, including dose, fractionation, irradiated volume, type of irradiation, co-administration of systemic therapy, size of the trial, as well as year it started. The impact of postponing radiation was not evaluated as such. Overall, it is, therefore, unclear which factors related to radiotherapy, if any, are detrimental to survival. We performed a critical review of the literature to assess the evidence on delay to radiation and outcome of breast cancer.

\section{Materials and methods}

Papers included in this review were identified by a computerized search of the Medline, Cancerlit and HealthStar databases from 1985 until the end of the year 2000, using as key words: breast neoplasms, radiotherapy, waiting lists, time factors, treatment delay, delay, waiting time, time interval, chemotherapy and time interval, combined modality therapy and time interval. Referenced articles in relevant papers were also reviewed. The search was restricted to English or French publications. Studies published only as abstracts were excluded. In addition, the Web sites of the United States National Cancer Institute and of the National Cancer Institute of Canada were visited for additional information on adjuvant therapy of breast cancer. The review excluded studies reporting only cosmetic outcomes of treatment.
Because these studies are heterogeneous, in particular with respect to the confounding effect of cotreatments, they were grouped according to whether or not chemotherapy was administered. Whenever possible, data were also provided separately for women having breast conserving surgery and total mastectomy. When start of follow-up was not explicitly specified, it was assumed to be the date of surgery. In addition, information on prognostic factors likely to influence outcomes of radiation therapy, including co-treatments, was taken into account when it was specified. Finally, periods of accrual and details of treatment were noted.

\section{Results}

Timing of radiotherapy was assessed in two groups of studies: studies on sequencing of chemotherapy and radiotherapy, and studies of delay to radiation given a specific chronological order of treatments.

\section{Sequencing of adjuvant treatment and outcomes of breast cancer}

Table 1 describes studies on sequencing of adjuvant radiotherapy and chemotherapy where patients treated by radiotherapy first were compared to those treated with chemotherapy followed by radiation. Studies on concomitant administration of both treatment modalities were excluded since they provide no information about delay. The earliest report was published by Recht et al. [10] who retrospectively reviewed 295 node-positive patients treated at the Joint Center for Radiation Therapy from 1976 to 1985. The radiation dose to the total breast was usually 45$46 \mathrm{~Gy}$, delivered at 1.8-2.0 Gy fractions five times weekly. The median total dose to the primary tumor site was $66 \mathrm{~Gy}$ and the nodal region was treated in $86 \%$ of cases. Chemotherapy consisted of CMF-based or doxorubicin-based regimens, alone or in combination. No patient received hormonal therapy (HT). The sequencing was: radiation followed by chemotherapy (RT-first) in 99 patients (34\%), sandwich therapy with chemotherapy administered both before and after irradiation in 54 patients (18\%), and concurrent radiotherapy and chemotherapy in 166 cases (39\%). Only 26 patients $(9 \%)$ had all chemotherapy completed before radiotherapy (CT-first). The CT-first group had the highest actuarial 5-year rate of local recurrences, $41 \%$ versus $4 \%$ in the RT-first group ( $p=0.03$ ), $8 \%$ in 
Table 1. Studies of the sequencing of chemotherapy and radiotherapy and treatment failure following primary treatment

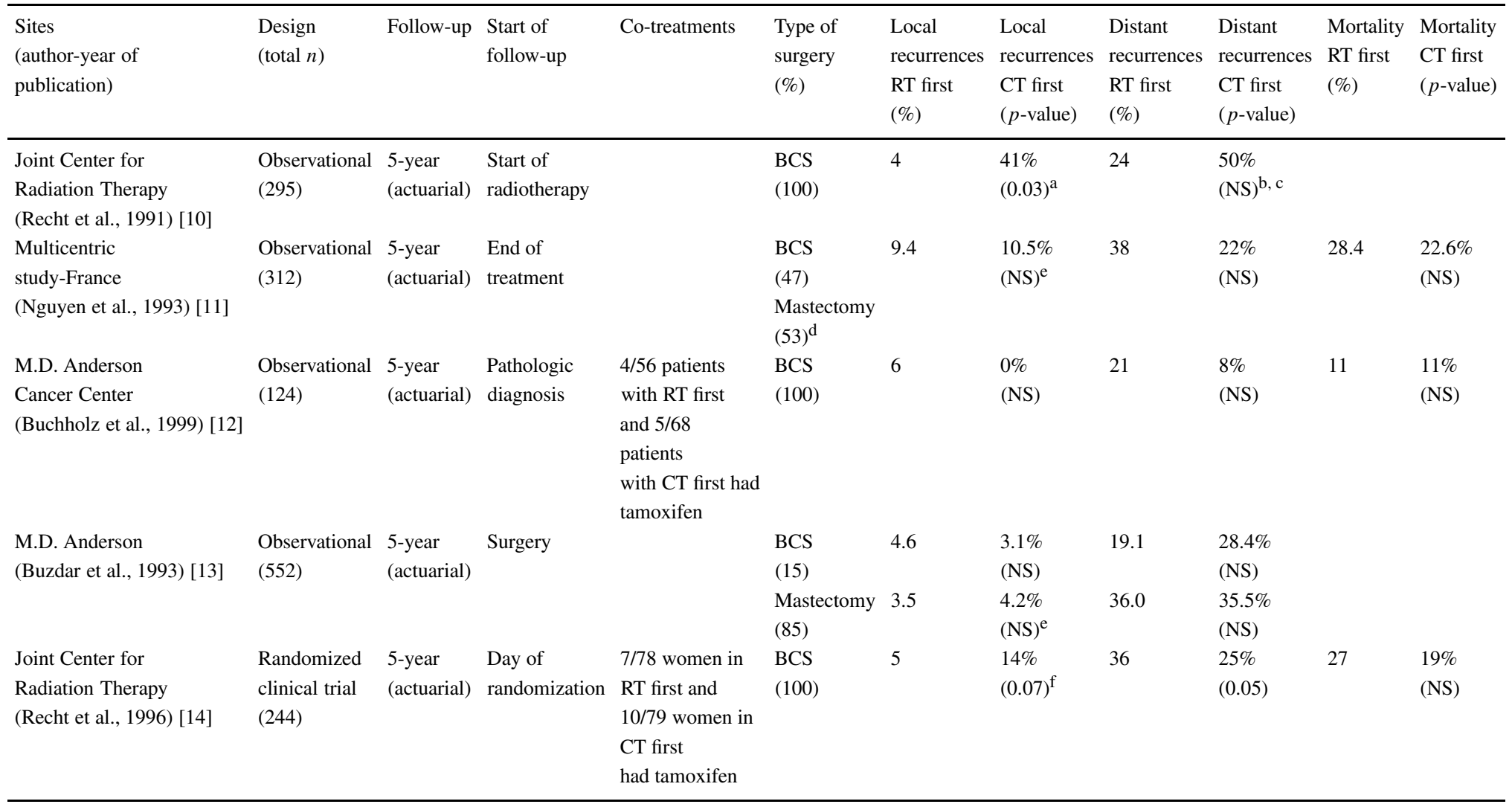

${ }^{a}$ All CT first. Part CT-RT-part CT: 8\% ( $p$-value all CT first versus CT-RT-CT: 0.04), CT-RT concurrent: $6 \%$ ( $p=0.06$ )

${ }^{b}$ Among women with $1-3$ positive nodes, age $\leq 50$ years and no doxorubicin received.

${ }^{c}$ All CT first. Part CT-RT-part CT: 11\% (NS), CT-RT concurrent: $18 \%$ (NS).

${ }^{\mathrm{d}}$ Results not available by type of surgical intervention.

${ }^{\mathrm{e}}$ Locoregional recurrences.

${ }^{\mathrm{f}}$ Crude rates. 
those with sandwich treatment $(p=0.04)$, and $6 \%$ in women receiving concomitant therapy $(p=0.06)$. For distant failures, these proportions were $50 \%$ (CT-first), $24 \%$ (RT-first), $11 \%$ (sandwich treatment), and $18 \%$ (concomitant therapy) ( $p$-value $=0.56$ for all groups being the same). For patients who received radiation therapy within 16 weeks of surgery, the 5-year actuarial local recurrence rate was 5\% compared with $35 \%$ in those beginning therapy more than 16 weeks after surgery $(p=0.0001)$. There also was a small but not statistically significant difference in the distant failure rate between women starting chemotherapy within 8 weeks of axillary dissection, $16 \%$, and those starting it later, $22 \%$. This study suggests that delaying radiation therapy may increase the likelihood of local failure, but it is limited by the small number of patients, especially in the radiation therapy first group, and the lack of details on factors likely to influence local recurrences, especially margin status. In addition, confounding by indication is likely to have influenced the results since the treatment decision was made by the medical and radiation oncologists in consultation, and no multivariate analysis was performed to adjust for differences in prognostic factors across groups.

In a multicentric study conducted in France, Nguyen et al. [11] assessed outcomes of breast cancer in 312 node-positive patients treated between 1977 and 1987, and followed for a minimum of 3 years. All were treated by surgery, radiation therapy, and multiagent chemotherapy. The surgical intervention was modified radical mastectomy in 53\% and tumorectomy in $47 \%$, but results were not reported separately for the two groups. Radiation therapy was delivered at a dose of 45-55 Gy in four or five fractions each week. A boost dose of 5-15 Gy was delivered to $15 \%$ of cases, and $42 \%$ had axillary irradiation. Group I included 125 patients who received radiotherapy immediately following surgery and group II, 187 with radiation delayed between 2 and more than 6 months after chemotherapy. A third of patients received CMF, the others an anthracycline-based regimen. The 5year actuarial rate of locoregional failure was $9.4 \%$ in group I and $10.5 \%$ in group II. For distant metastasis, the rates were 38 and $22 \%$, whereas survival was estimated at 71.6 and $77.4 \%$, respectively. These differences were not significant, and no multivariate analysis was performed to account for differences in prognostic factors between groups.

Two more recent observational studies were conducted at the University of Texas M.D. Anderson Cancer Center during overlapping periods of time.
The first one, by Buchholz et al. [12], evaluated chemotherapy and radiation sequencing in 124 nodenegative patients treated with breast- conserving surgery between 1982 and 1996. Only patients who received their radiation at this institution were included. The two groups of patients were similar in age, tumor stage, margin status, as well as hormone receptor status. The median follow-up was 44 months for the chemotherapy-first group and 61 months for the radiation-first group. The radiation treatment was given using opposed tangential fields to a median dose of $50 \mathrm{~Gy}$. The majority of women had a boost to the primary tumor bed. Doxorubicin-based combination therapy with a median of six cycles was most often used. Less than $10 \%$ of women in each group also received tamoxifen. There were no significant differences in outcomes for any of the endpoints studied but the statistical power was limited. The local control rates were 100 and $94 \%$ in the CT-first and RT-first groups, whereas the distant control rates were 92 and $79 \%$, and overall survival at 5 years was $89 \%$ in each group. The second review by Buzdar et al. [13] included 552 patients with both node-negative and positive disease treated between 1974 and 1990 with either total mastectomy $(n=467)$ or segmental mastectomy $(n=85)$, irradiation, and combination chemotherapy. Within each surgical modality, patients were classified according to whether chemotherapy or radiation therapy was administered first. As in the previous series, chemotherapy usually consisted of doxorubicin-containing regimens (FAC or VACP), sometimes with modifications. Details of irradiation protocols were not reported but are likely to be similar in both reviews. Delay to irradiation in women receiving chemotherapy first was at least 20 weeks. A higher proportion of women in the chemotherapy first group had more than four nodes invaded ( $84 \%$ versus $58 \%$, $p<0.01)$. Among women having total mastectomy, the 5-year estimated recurrence rates in the group with chemotherapy first as compared with the group with radiation therapy first were $4.2 \% \pm 3 \%$ versus $3.5 \% \pm 1 \%, p=0.44$ for locoregional recurrences, and $35.5 \% \pm 7 \%$ versus $36.0 \% \pm 2.7 \%, p=0.80$, for systemic recurrences. Adjustment for age and number of involved nodes did not modify these associations. Among women treated with segmental mastectomy, more women in the chemotherapy first group were ER-positive $(54 \%$ versus $30 \%, p=0.05)$. The rates of both locoregional recurrences and systemic recurrences were similar in the two groups $(3.1 \% \pm 3 \%$ versus $4.6 \% \pm 3 \%, p=0.76 ; 28.4 \% \pm 13 \%$ versus 
$19.1 \% \pm 7 \%, p=0.67)$. Conclusions from these two studies are consistent and suggest that regardless of the type of surgery and extent of disease, the chemotherapy-radiation therapy sequence does not influence the local or systemic recurrence rates. Although observational, these studies at least partially adjusted for significant covariates likely to influence outcomes. The independence of the samples is unclear.

The only published randomized clinical trial evaluating the sequencing of chemotherapy and radiation therapy after conservative surgery for early-stage breast cancer was conducted in Boston, and included 244 patients diagnosed with stage I or II breast cancer treated with wide local excision between 1984 and 1992 [14]. Less than $15 \%$ were node-negative. They received a 12-week course of adjuvant chemotherapy with methotrexate, leucovorin, fluorouracil, cyclophosphamide, prednisone, and doxorubicin, administered either before (CT-first group) or after irradiation (RT-first group). Radiation therapy was delivered to the entire breast to a dose of $45 \mathrm{~Gy}$ in 25 fractions, followed by a boost of $16-18 \mathrm{~Gy}$, and 36 and $35 \%$ of patients in the RT and CT-first groups, respectively received nodal irradiation. Both groups were similar in prognostic factors (age, lymphatic invasion, ER, margins, and nodal status), except that a larger proportion of patients in the RT-first group had tumors with an extensive intraductal component. After completion of both radiotherapy and chemotherapy, tamoxifen was given, starting in 1988, to seven patients in the RT-first group and 10 in the CT-first group. The median interval between last surgery and radiation therapy was 36 and 126 days, respectively. For chemotherapy, it was 119 and 52 days, respectively. The 5-year actuarial rates of cancer recurrence at any site, distant metastases, and survival in women with RT-first and CT-first were 38 and $31 \%(p=0.17)$, 36 and $25 \%(p=0.05)$, and 73 and $81 \%(p=0.11)$. There was a difference in the pattern of recurrences of borderline significance, the 5-year crude rates of local recurrence being 5 and $14 \%$, and for regional or distant recurrences, 32 and $20 \%(p=0.07)$. Sequence assignment, invasion of lymphatic vessels, and margin re-excision all had significant effects on the risks of recurrence at various sites in multivariate analysis and interaction of sequence with margin status and number of invaded axillary nodes was significant. In patients with negative margins, there was little influence of treatment sequence whereas in those with close, positive or unknown margins, the higher incidence of local recurrence in the CT-first group and higher incidence of distant metastasis in the RT-first group persisted ( $p$-value for sequence interaction $<0.001$ ). Similarly, in patients with one to three positive nodes, the sequence assignment made little difference, but for those with either negative or four or more positive nodes, more local recurrences were found in the CT-first group and more distant recurrences in the RT-first group. Overall, this study suggests that patients at high risk of recurrence should receive chemotherapy before radiation. Because of the long time of accrual and small sample size, it is vulnerable to some biases, as a result of changes in medical care over this period and risk of both $\beta$, and to a lower extent $\alpha$, errors.

\section{Time to radiation and outcomes of breast cancer}

Studies on delay to adjuvant radiation therapy and outcomes of breast cancer are summarized in Tables 2 and 3. They were grouped according to whether or not systemic treatment with chemotherapy was given in addition to surgery and radiotherapy. Two randomized trials of chemotherapy provide some information on delay to radiation among women having breast conserving surgery and are included. All other studies are observational retrospective case series.

\section{Time to radiation and outcomes of breast cancer in studies restricted to women not receiving chemotherapy}

Four studies of the impact of delaying radiation therapy following surgery among women not receiving chemotherapy are summarized in Table 2 [15-21]. In all but one [18], tamoxifen was not given either. All four were restricted to women having breast conserving surgery and adjusted for significant prognostic factors. Although they used different time frames for the definition of the treatment groups and followed their study populations for different periods of time, their conclusions are remarkably similar.

The earliest series came from the Institut GustaveRoussy and included 436 patients with stage T1 and small T2 breast carcinoma, all treated between 1970 and 1981 with breast-conserving surgery and radiotherapy [15]. Surgery consisted of excisional biopsy or quadrantectomy when the tumor size was $20 \mathrm{~mm}$ or less (1970-1979), or equal to or less than $25 \mathrm{~mm}$ (1980-81). Eight patients with tumor size greater than $25 \mathrm{~mm}$ who refused mastectomy were included in the study. Radiotherapy was delivered using a Cobalt unit to a dose of $45 \mathrm{~Gy}$, in $2.5 \mathrm{~Gy}$ fractions, four times weekly to the breast, and when indicated, to the re- 
Table 2. Studies of surgery-radiotherapy interval and treatment failure following primary treatment among women without chemotherapy

\begin{tabular}{|c|c|c|c|c|c|c|c|c|c|c|c|c|}
\hline $\begin{array}{l}\text { Sites } \\
\text { (author-year of } \\
\text { publication) }\end{array}$ & $\begin{array}{l}\text { Design } \\
(\text { total } n \text { ) }\end{array}$ & $\begin{array}{l}\text { Time } \\
\text { intervals to } \\
\text { RT }\end{array}$ & Follow-up & $\begin{array}{l}\text { Start of } \\
\text { follow-up }\end{array}$ & Co-treatments & $\begin{array}{l}\text { Type of } \\
\text { surgery } \\
(\%)\end{array}$ & $\begin{array}{l}\text { Local } \\
\text { recurrences } \\
\text { early RT } \\
(\%)\end{array}$ & $\begin{array}{l}\text { Local } \\
\text { recurrences } \\
\text { late } \mathrm{RT} \\
(p \text {-value) }\end{array}$ & $\begin{array}{l}\text { Distant } \\
\text { recurrences } \\
\text { early RT } \\
(\%)\end{array}$ & $\begin{array}{l}\text { Distant } \\
\text { recurrences } \\
\text { late RT } \\
\text { (p-value) }\end{array}$ & $\begin{array}{l}\text { Mortality } \\
\text { early RT }\end{array}$ & $\begin{array}{l}\text { Mortality } \\
\text { late RT } \\
\text { ( } p \text {-value) }\end{array}$ \\
\hline $\begin{array}{l}\text { Institut Gustave- } \\
\text { Roussy } \\
\text { (Clarke et al., 1985) [15] }\end{array}$ & $\begin{array}{l}\text { Observational } \\
(436)\end{array}$ & $\begin{array}{l}<7 \text { weeks, } \\
\geq 7 \text { weeks }\end{array}$ & $\begin{array}{l}\text { 10-year } \\
\text { (actuarial) }\end{array}$ & Surgery & & $\begin{array}{l}\text { BCS } \\
(100)\end{array}$ & 4.5 & $\begin{array}{l}12.1 \% \\
(0.01)^{\mathrm{a}}\end{array}$ & & & & \\
\hline $\begin{array}{l}\text { London Regional } \\
\text { Cancer Center } \\
\text { (Vujovic et al., 1998) [17] }\end{array}$ & $\begin{array}{l}\text { Observational } \\
(568)\end{array}$ & $\begin{array}{l}\leq 12 \text { weeks } \\
>12 \text { weeks }\end{array}$ & $\begin{array}{l}\text { 5-year } \\
\text { (actuarial) }\end{array}$ & Surgery & & $\begin{array}{l}\text { BCS } \\
(100)\end{array}$ & 7.8 & $\begin{array}{l}3.8 \% \\
(\mathrm{NS})^{\mathrm{c}}\end{array}$ & & & & \\
\hline $\begin{array}{l}\text { British Columbia } \\
\text { Cancer Agency } \\
\text { (Froud et al., 2000) [18] }\end{array}$ & $\begin{array}{l}\text { Observational } \\
\text { (1962) }\end{array}$ & $\begin{array}{l}<6 \text { weeks, } \\
6-8 \text { weeks, } \\
9-12 \text { weeks, } \\
\geq 13 \text { weeks }\end{array}$ & $\begin{array}{l}\text { 5-year } \\
\text { (actuarial) }\end{array}$ & Surgery & $\begin{array}{l}677 / 1962 \\
\text { patients } \\
\text { received } \\
\text { tamoxifen }\end{array}$ & $\begin{array}{l}\text { BCS } \\
(100)\end{array}$ & $\begin{array}{l}3.3,2.2, \\
2.8\end{array}$ & $\begin{array}{l}2.4 \% \\
\text { (NS) }\end{array}$ & $\begin{array}{l}9.6,7.9, \\
8.5\end{array}$ & $\begin{array}{l}14.0 \% \\
(0.027)^{\mathrm{e}}\end{array}$ & & \\
\hline
\end{tabular}

${ }^{a}$ Locoregional recurrences.

Statistical comparison limited to 0-4 weeks and 5-8 weeks.

${ }^{c}$ Crude rates.

${ }^{\mathrm{d}}$ Distant or regional recurrences.

${ }^{\mathrm{e}} 13+$ versus $0-5$ weeks.

(3) vers 
Table 3. Studies of surgery-radiotherapy interval and treatment failure following primary treatment among women given chemotherapy

\begin{tabular}{|c|c|c|c|c|c|c|c|c|c|c|c|c|}
\hline $\begin{array}{l}\text { Sites } \\
\text { (author-year of } \\
\text { publication) }\end{array}$ & $\begin{array}{l}\text { Design } \\
(\text { total } n \text { ) }\end{array}$ & $\begin{array}{l}\text { Time intervals } \\
\text { to } \\
\text { RT }\end{array}$ & Follow-up & $\begin{array}{l}\text { Start of } \\
\text { follow-up }\end{array}$ & $\begin{array}{l}\text { Co- } \\
\text { treatments }\end{array}$ & $\begin{array}{l}\text { Type of } \\
\text { surgery } \\
(\%)\end{array}$ & $\begin{array}{l}\text { Local } \\
\text { recurrences } \\
\text { early RT } \\
(\%)\end{array}$ & $\begin{array}{l}\text { Local } \\
\text { recurrences } \\
\text { late } \mathrm{RT} \\
\text { ( } p \text {-value) }\end{array}$ & $\begin{array}{l}\text { Distant } \\
\text { recurrences } \\
\text { early RT } \\
(\%)\end{array}$ & $\begin{array}{l}\text { Distant } \\
\text { recurrences } \\
\text { late RT } \\
\text { ( } p \text {-value) }\end{array}$ & $\begin{array}{l}\text { Mortality } \\
\text { early RT } \\
(\%)\end{array}$ & $\begin{array}{l}\text { Mortality } \\
\text { late RT } \\
\text { ( } p \text {-value) }\end{array}$ \\
\hline $\begin{array}{l}\text { Joint Center for } \\
\text { Radiation Therapy } \\
\text { (Recht et al., 1991) [10] }\end{array}$ & $\begin{array}{l}\text { Observational } \\
(295)\end{array}$ & $\begin{array}{l}\leq 16 \text { weeks, } \\
>16 \text { weeks }\end{array}$ & $\begin{array}{l}\text { 5-year } \\
\text { (actuarial) }\end{array}$ & $\begin{array}{l}\text { Start of } \\
\text { radiotherapy }\end{array}$ & $\begin{array}{l}295 / 295 \\
\text { patients } \\
\text { received CT }\end{array}$ & $\begin{array}{l}\text { BCS } \\
(100)\end{array}$ & 5 & $\begin{array}{l}35 \% \\
(0.0001)\end{array}$ & & & & \\
\hline $\begin{array}{l}\text { National Surgical } \\
\text { Adjuvant Breast and } \\
\text { Bowel Project } \\
\text { (Fisher et al., 1990) [19] }\end{array}$ & $\begin{array}{l}\text { Randomized } \\
\text { clinical trial } \\
\text { NSABP B-15 } \\
(595)\end{array}$ & $\begin{array}{l}\text { 6-9 weeks, } \\
14-17 \text { weeks }\end{array}$ & $\begin{array}{l}\text { 3-year } \\
\text { (actuarial) }\end{array}$ & Surgery & $\begin{array}{l}595 / 595 \\
\text { patients } \\
\text { received CT }\end{array}$ & $\begin{array}{l}\text { BCS } \\
(100)\end{array}$ & & & & & $14^{\mathrm{a}}$ & $\begin{array}{l}10 \%, \\
16 \%{ }^{\mathrm{a}} \\
\text { (NS) }\end{array}$ \\
\hline $\begin{array}{l}\text { International Breast } \\
\text { Cancer Study Group } \\
\text { (Wallgren et al., 1996) [20] }\end{array}$ & $\begin{array}{l}\text { Randomized } \\
\text { clinical trial } \\
\text { (IBCSG VI) } \\
(433)\end{array}$ & $\begin{array}{l}\text { Medians of } 4.0 \\
\text { and } 6.6 \text { months }\end{array}$ & $\begin{array}{l}\text { 4-year } \\
\text { (actuarial) }\end{array}$ & $\begin{array}{l}\text { Day of } \\
\text { randomization }\end{array}$ & $\begin{array}{l}433 / 433 \\
\text { patients } \\
\text { received CT }\end{array}$ & $\begin{array}{l}\text { BCS } \\
(100)\end{array}$ & 8 & $\begin{array}{l}7 \% \\
(\mathrm{NS})^{\mathrm{b}, \mathrm{c}}\end{array}$ & 18 & $\begin{array}{l}23 \% \\
\text { (NS) }\end{array}$ & 10 & $\begin{array}{l}16 \% \\
\text { (NS) }\end{array}$ \\
\hline $\begin{array}{l}\text { International Breast } \\
\text { Cancer Study Group } \\
\text { (Wallgren et al., 1996) [20] }\end{array}$ & $\begin{array}{l}\text { Randomized } \\
\text { clinical trial } \\
\text { (IBCSG VII) } \\
(285)\end{array}$ & $\begin{array}{l}\text { Medians of } 2.3 \\
\text { and } 3.9 \text { months }\end{array}$ & $\begin{array}{l}\text { 4-year } \\
\text { (actuarial) }\end{array}$ & $\begin{array}{l}\text { Day of } \\
\text { randomization }\end{array}$ & $\begin{array}{l}285 / 285 \\
\text { patients } \\
\text { received } \\
\text { tamoxifen } \\
139 / 285 \\
\text { patients } \\
\text { received CT }\end{array}$ & $\begin{array}{l}\text { BCS } \\
(100)\end{array}$ & 3 & $\begin{array}{l}6 \\
(\mathrm{NS})^{\mathrm{b}, \mathrm{c}}\end{array}$ & 18 & $\begin{array}{l}15 \% \\
(\mathrm{NS})\end{array}$ & 11 & $\begin{array}{l}11 \% \\
\text { (NS) }\end{array}$ \\
\hline
\end{tabular}


Table 3. (continued)

\begin{tabular}{|c|c|c|c|c|c|c|c|c|c|c|c|c|}
\hline $\begin{array}{l}\text { Sites } \\
\text { (author-year of } \\
\text { publication) }\end{array}$ & $\begin{array}{l}\text { Design } \\
\text { (total } n \text { ) }\end{array}$ & $\begin{array}{l}\text { Time } \\
\text { intervals to } \\
\text { RT }\end{array}$ & Follow-up & $\begin{array}{l}\text { Start of } \\
\text { follow-up }\end{array}$ & $\begin{array}{l}\text { Co- } \\
\text { treatments }\end{array}$ & $\begin{array}{l}\text { Type of } \\
\text { surgery } \\
(\%)\end{array}$ & $\begin{array}{l}\text { Local } \\
\text { recurrences } \\
\text { early RT } \\
(\%)\end{array}$ & $\begin{array}{l}\text { Local } \\
\text { recurrences } \\
\text { late } \mathrm{RT} \\
\text { ( } p \text {-value) }\end{array}$ & $\begin{array}{l}\text { Distant } \\
\text { recurrences } \\
\text { early RT } \\
(\%)\end{array}$ & $\begin{array}{l}\text { Distant } \\
\text { recurrences } \\
\text { late RT } \\
\text { ( } p \text {-value) }\end{array}$ & $\begin{array}{l}\text { Mortality } \\
\text { early RT } \\
(\%)\end{array}$ & $\begin{array}{l}\text { Mortality } \\
\text { late RT } \\
\text { ( } p \text {-value) }\end{array}$ \\
\hline $\begin{array}{l}\text { Memorial Sloan- } \\
\text { Kettering Cancer } \\
\text { Center } \\
\text { (McCormick } \\
\text { et al., 1996) [21] }\end{array}$ & $\begin{array}{l}\text { Observational } \\
(471)\end{array}$ & $\begin{array}{l}\text { Means of } \\
6 \text { weeks, } \\
7 \text { weeks, } \\
29 \text { weeks }\end{array}$ & $\begin{array}{l}\text { 5-year } \\
\text { (actuarial) }\end{array}$ & Surgery & $\begin{array}{l}139 / 471 \\
\text { patients } \\
\text { received CT }\end{array}$ & $\begin{array}{l}\text { BCS } \\
(100)\end{array}$ & 4 & $\begin{array}{l}5 \%, 14 \% \\
(\mathrm{NS})^{\mathrm{d}, \mathrm{e}}\end{array}$ & & & & \\
\hline $\begin{array}{l}\text { University of } \\
\text { Washington } \\
\text { Medical Center } \\
\text { (Buchholz } \\
\text { et al., 1993) [22] }\end{array}$ & $\begin{array}{l}\text { Observational } \\
\text { (105) }\end{array}$ & $\begin{array}{l}<6 \text { months, } \\
\geq 6 \text { months }\end{array}$ & $\begin{array}{l}\text { 8-year } \\
\text { (actuarial) }\end{array}$ & Diagnosis & $\begin{array}{l}105 / 105 \\
\text { patients } \\
\text { received CT }\end{array}$ & $\begin{array}{l}\text { BCS } \\
(60) \\
\text { Mastectomy } \\
(40)\end{array}$ & $\begin{array}{l}0 \\
5.3\end{array}$ & $\begin{array}{l}19.8 \% \\
(0.020) \\
23 \% \\
(\mathrm{NS})\end{array}$ & & & $\begin{array}{l}3.8 \\
30.1\end{array}$ & $\begin{array}{l}51.7 \% \\
(0.007) \\
48 \% \\
(\mathrm{NS})\end{array}$ \\
\hline $\begin{array}{l}\text { Rush-Presbyterian- } \\
\text { St.Luke's Medical } \\
\text { Center } \\
\text { (Hartsell } \\
\text { et al., 1995) [23] }\end{array}$ & $\begin{array}{l}\text { Observational } \\
\text { (84) }\end{array}$ & $\begin{array}{l}<120 \text { days } \\
\geq 120 \text { days }\end{array}$ & $\begin{array}{l}\text { 5-year } \\
\text { (actuarial) }\end{array}$ & Surgery & $\begin{array}{l}84 / 84 \\
\text { patients } \\
\text { received CT }\end{array}$ & $\begin{array}{l}\text { BCS } \\
(100)\end{array}$ & 2 & $\begin{array}{l}14 \% \\
(0.05)\end{array}$ & & & 13 & $\begin{array}{l}18 \% \\
\text { (NS) }\end{array}$ \\
\hline $\begin{array}{l}\text { Swedish Medical } \\
\text { Center } \\
\text { (Leonard } \\
\text { et al., 1995) [24] }\end{array}$ & $\begin{array}{l}\text { Observational } \\
\text { (262) }\end{array}$ & $\begin{array}{l}<6 \text { months, } \\
\leq 6 \text { months }\end{array}$ & $\begin{array}{l}\text { 5-year } \\
\text { (actuarial) }\end{array}$ & Surgery & $\begin{array}{l}153 / 262 \\
\text { patients } \\
\text { received } \\
\text { tamoxifen } \\
105 / 262 \\
\text { patients } \\
\text { received } \\
\text { CT }\end{array}$ & $\begin{array}{l}\text { BCS } \\
(100)\end{array}$ & $\begin{array}{l}\text { Group I: } 5 \\
\text { Group II: } 5\end{array}$ & $\begin{array}{l}\text { Group I: } 2 \% \\
\text { Group II: } 0 \% \\
\text { (NS) }\end{array}$ & & & $\begin{array}{l}\text { Group I: } 5 \\
\text { Group II: } 16\end{array}$ & $\begin{array}{l}\text { Group I: } \\
5 \% \\
\text { Group II: } \\
0 \% \\
\text { (NS) }\end{array}$ \\
\hline $\begin{array}{l}\text { University Hospital, } \\
\text { Stony Brook, NY } \\
\text { (Meek et al., } \\
\text { 1996) [25] }\end{array}$ & $\begin{array}{l}\text { Observational } \\
(310)\end{array}$ & $\begin{array}{l}2-59 \text { weeks, } \\
\text { mean of } \\
8 \text { weeks, } \\
12-63 \text { weeks, } \\
\text { mean of } 31 \\
\text { weeks }\end{array}$ & $\begin{array}{l}\text { 10-year } \\
\text { (actuarial) }\end{array}$ & $\begin{array}{l}\text { Pathological } \\
\text { diagnosis }\end{array}$ & $\begin{array}{l}102 / 310 \\
\text { cases } \\
\text { received } \\
\text { tamoxifen } \\
76 / 310 \text { cases } \\
\text { received } \\
\text { CT }\end{array}$ & $\begin{array}{l}\text { BCS } \\
(100)\end{array}$ & 4 & $\begin{array}{l}2 \% \\
\text { (NS) }\end{array}$ & 15 & $\begin{array}{l}8 \% \\
\text { (NS) }\end{array}$ & 7.3 & $\begin{array}{l}4.8 \% \\
(\mathrm{NS})^{\mathrm{f}}\end{array}$ \\
\hline
\end{tabular}


Table 3. (continued)

\begin{tabular}{|c|c|c|c|c|c|c|c|c|c|c|c|c|}
\hline $\begin{array}{l}\text { Sites } \\
\text { (author-year of } \\
\text { publication) }\end{array}$ & $\begin{array}{l}\text { Design } \\
(\text { total } n \text { ) }\end{array}$ & $\begin{array}{l}\text { Time } \\
\text { intervals to } \\
\text { RT }\end{array}$ & Follow-up & $\begin{array}{l}\text { Start of } \\
\text { follow-up }\end{array}$ & $\begin{array}{l}\text { Co- } \\
\text { treatments }\end{array}$ & $\begin{array}{l}\text { Type of } \\
\text { surgery } \\
(\%)\end{array}$ & $\begin{array}{l}\text { Local } \\
\text { recurrences } \\
\text { early RT } \\
(\%)\end{array}$ & $\begin{array}{l}\text { Local } \\
\text { recurrences } \\
\text { late RT } \\
(p \text {-value) }\end{array}$ & $\begin{array}{l}\text { Distant } \\
\text { recurrences } \\
\text { early RT } \\
(\%)\end{array}$ & $\begin{array}{l}\text { Distant } \\
\text { recurrences } \\
\text { late RT } \\
(p \text {-value) }\end{array}$ & $\begin{array}{l}\text { Mortality } \\
\text { early RT } \\
(\%)\end{array}$ & $\begin{array}{l}\text { Mortality } \\
\text { late RT } \\
\text { ( } p \text {-value) }\end{array}$ \\
\hline \multirow[t]{2}{*}{$\begin{array}{l}\text { Louisiana State } \\
\text { University } \\
\text { School of } \\
\text { Medicine } \\
\text { (Ampil } \\
\text { et al., 1999) [26] }\end{array}$} & $\begin{array}{l}\text { Observational } \\
\text { (47) }\end{array}$ & $\begin{array}{l}\text { Group I: } \leq 7 \\
\text { weeks, }>7 \\
\text { weeks } \\
\text { Group II: } \leq 24 \\
\text { weeks, }>24 \\
\text { weeks }\end{array}$ & $\begin{array}{l}\text { 4-year } \\
\text { (crude) }\end{array}$ & Diagnosis & $\begin{array}{l}\text { Group I: } \\
\text { 0/26 patients } \\
\text { received CT }\end{array}$ & $\begin{array}{l}\text { BCS } \\
(100)\end{array}$ & Group I: 0 & $\begin{array}{l}\text { Group I: } 18 \% \\
\text { (NS) }\end{array}$ & Group I: 0 & $\begin{array}{l}\text { Group I: } \\
12 \% \\
\text { (NS) }\end{array}$ & Group I: 22 & $\begin{array}{l}\text { Group I: } 71 \% \\
(0.05)\end{array}$ \\
\hline & & & & & $\begin{array}{l}\text { Group II: } \\
21 / 21 \\
\text { patients } \\
\text { received CT }\end{array}$ & & Group II: 0 & $\begin{array}{l}\text { Group II: } 11 \% \\
\text { (NS) }\end{array}$ & Group II: 0 & $\begin{array}{l}\text { Group II: } \\
6 \% \\
(\mathrm{NS})\end{array}$ & Group II: 67 & $\begin{array}{l}\text { Group II: } 61 \% \\
\text { (NS) }\end{array}$ \\
\hline $\begin{array}{l}\text { University of } \\
\text { Pennsylvania } \\
\text { (Metz } \\
\text { et al., 2000) [27] }\end{array}$ & $\begin{array}{l}\text { Observational } \\
(221)\end{array}$ & $\begin{array}{l}\leq 2 \text { months, } \\
2.1-6 \text { months, } \\
>6 \text { months }\end{array}$ & $\begin{array}{l}\text { 8-year } \\
\text { (actuarial) }\end{array}$ & $\begin{array}{l}\text { Radiation } \\
\text { therapy }\end{array}$ & $\begin{array}{l}151 / 221 \\
\text { patients } \\
\text { received CT } \\
116 / 221 \\
\text { patients } \\
\text { received HT } \\
79 / 221 \\
\text { patients } \\
\text { received } \\
\text { both CT and } \\
\text { HT }\end{array}$ & $\begin{array}{l}\text { Mastectomy } \\
(100)\end{array}$ & 13 & $\begin{array}{l}4 \%, 12 \% \\
\text { (NS) })^{g, h}\end{array}$ & & & & \\
\hline $\begin{array}{l}\text { Free University } \\
\text { Hospital, The } \\
\text { Netherlands } \\
\text { (Slotman } \\
\text { et al., 1994) [28] }\end{array}$ & $\begin{array}{l}\text { Observational } \\
(508)\end{array}$ & $\begin{array}{l}<50 \text { days } \\
\geq 50 \text { days }\end{array}$ & $\begin{array}{l}\text { 5-year } \\
\text { (actuarial) }\end{array}$ & Surgery & $\begin{array}{l}73 / 508 \\
\text { patients } \\
\text { received CT } \\
92 / 508 \\
\text { patients } \\
\text { received } \\
\text { tamoxifen }\end{array}$ & $\begin{array}{l}\text { BCS } \\
(100)\end{array}$ & 1.7 & $\begin{array}{l}5.6 \% \\
(<0.05)^{\mathrm{h}}\end{array}$ & & (NS) & & (NS) \\
\hline
\end{tabular}

a Percentages estimated from Figure 2 in [19].

${ }^{\mathrm{b}}$ Four-year crude rates of local failure with or without other sites.

${ }^{\mathrm{c}} \mathrm{Based}$ on patients with at least 4 years of follow-up who received radiation therapy.

${ }^{\mathrm{d} C o m p a r i s o n}$ involves all three groups.

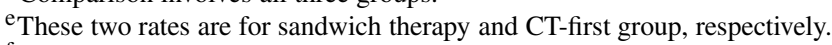

${ }_{\mathrm{f}}^{\mathrm{f}}$ Five-year risk of death with distant disease.

g Locoregional recurrences.

$\mathrm{h}$ Rates are for the whole study population. 
gional lymph nodes. A $15 \mathrm{~Gy}$ boost to the tumor bed was routinely administered at the same fractionation, and axillary or internal mammary node boosts of 1015 Gy were occasionally delivered. The 5- and 10-year actuarial locoregional control rates were 93 and $90 \%$, respectively. Time to irradiation was a significant predictor of both breast and locoregional recurrences in univariate analysis, the relative risk of locoregional relapse for women receiving this treatment seven or more weeks after their excisional biopsy being 3.3, $p=0.01$, as compared to those receiving it earlier. There was no difference between treatment intervals of 7-8 versus 9 or more weeks. Time to irradiation, however, lost its significance as a prognostic factor in multivariate analysis that also included tumor grade and radiation dose to the entire breast. Margin status was not an independent predictor of locoregional recurrence in this study.

More recently, Nixon et al. [16] reviewed 653 patients with stage I and II node-negative breast cancer, treated between 1968 and 1985 at the Joint Center for Radiation Therapy in Boston. All underwent complete gross excision of their tumor and usually received a dose of 45-50 Gy to the tumor bed delivered over a period of approximately 5 weeks. This was followed by a boost to the tumor bed to bring the total minimum dose to $60 \mathrm{~Gy}$. Two hundred and eighty three women received radiation within 4 weeks of surgery, 308 between 5 and 8 weeks, and 54 started it 9-12 weeks after surgery. Statistical comparison was limited to the first two groups. Median follow-up was 100 months. Pathologic features were similar in patients treated 04 weeks and 5-8 weeks after surgery. There were no differences in their 5-year crude rates of local recurrences (13 and 7\%) or regional/distant recurrences (11 and $14 \%$ ). Young age, high grade, lymphatic vessel invasion, and absence of a mononuclear cellular reaction were significant predictors of recurrences, but not delay to irradiation in multivariate analysis (risk ratio $=0.89, p=0.44$ ).

Two recent investigations were conducted in Canada. The first [17] included 568 patients with T1-T2, N0 breast cancer treated by lumpectomy and radiation therapy between 1985 and 1992 in Ontario, Canada. Radiotherapy was administered to the whole breast using two different fractionation schedules. One was 50 Gy delivered in 25 fractions over 5 weeks, with a boost of 6-16 Gy in 3-8 fractions to women with close or positive margins. The other schedule consisted of $40 \mathrm{~Gy}$ in 15 or 16 fractions over 3-3.5 weeks to the whole breast, followed by a direct electron boost of $12.5 \mathrm{~Gy}$ in $4-5$ fractions for negative margins or $15 \mathrm{~Gy}$ in five fractions for positive margins. Median follow-up was 63.5 months. There were no differences in local recurrences or disease-free survival among women treated $0-8,8-12,12-16$, and more than 16 weeks after surgery. The crude local recurrence rate was $6.9 \%$; local recurrence was $7.8 \%$ for patients treated within 12 weeks, and $3.8 \%$ for those treated more than 12 weeks after surgery, a nonsignificant difference. Multivariate analysis showed that age, tumor size, and grade, but not the surgery-radiotherapy interval, were significant predictors of disease-free survival. Margin status was a significant predictor of local breast recurrence (risk ratio $=0.547, p=0.014$ ), but not disease-free survival.

The effect of time interval between breastconserving surgery and radiation therapy was also evaluated among 1962 women treated in British Columbia between 1989 and 1993 [18]. Subjects were less than 90-year-old at diagnosis, not stage T4 or $\mathrm{M} 1$, and had survived for 30 or more days from diagnosis. About $80 \%$ were node-negative and $4.5 \%$ had unknown nodal status. Tamoxifen was given to 677 patients $(34.5 \%)$. All patients received tangent pair RT to the whole breast, and $81.8 \%$ received $44 \mathrm{~Gy}$. A relatively short fractionation over 3 or 4 weeks was usually used, and $19.2 \%$ received a boost to the primary site. Cox proportional hazards regression models were fitted, adjusting for age and residence, pathologic factors (tumor size, grade, lymphatic, vascular or perineural invasion, level of estrogen receptors (ER), histology, nodal status, and margins), as well as use of tamoxifen and treatment center. Median follow-up was 71 months. No difference in ipsilateral breast recurrences was observed for any time interval to RT. The results were similar when the analysis was restricted to women not using tamoxifen. The cumulative incidence of systemic relapse was higher for patients with longer interval to RT (14\% for interval $13+$ weeks versus $9.6 \%$ for $0-5$ weeks, $p=0.027$ ). In multivariate analyses, however, this lost significance and only tumor grade, size, and nodal status remained significant predictors of systemic relapse, whereas grade and use of tamoxifen were associated with in-breast relapses. Resection margins were not associated with either outcome in this study.

Both of the Canadian studies involved large numbers of subjects followed for comparable periods of time, adjusted for significant covariates in multivariate models, and defined late radiation therapy within comparable time frames. 
Time to radiation and outcomes of breast cancer in studies including women receiving chemotherapy

Several studies have assessed outcomes of radiation therapy in study populations also receiving systemic treatment [19-28]. They are summarized in Table 3. Two of them are randomized trials of chemotherapy and provided data on subgroups of women having breast conserving surgery and radiotherapy according to schedules that varied with systemic treatment received, therefore offering the opportunity to study the impact of delaying irradiation $[19,20]$. In one trial [20], three or six cycles of CMF were administered, whereas in the other [19], women receiving four cycles of AC over 2 months with or without further therapy were compared to those receiving 6 months of CMF. In observational studies, chemotherapy consisted of CMF in one case [28], whereas in the others, either CMF or an anthracycline-based regimen was usually used [21-24, 26, 27]. Type of chemotherapy was unspecified in one report [25].

These investigations represent a mixed group, since in some cases [19, 20, 22, 23], all participants did receive chemotherapy, whereas in others, individuals receiving chemotherapy were compared to those not receiving systemic treatment [21]. In four studies [24, 26-28], analyses by subgroup of systemic therapy were done. Finally, in one case [25], a fraction of patients in each group received some systemic treatment, but data were not reported separately for this specific group and no multivariate analysis was performed to adjust for this factor.

Two reports are subset analyses of randomized clinical trials. The NSABP B-15 trial [19] was designed to compare four cycles of AC given in 2 months with or without further therapy with CMF to 6 months of conventional CMF. After January 1985, lumpectomy plus axillary dissection and radiation therapy became accepted care and women so treated became eligible, providing some information about the impact of delaying radiotherapy which was given after either four cycles of AC or one cycle of CMF. At 3 years of follow-up, there were no significant differences in either disease-free, distant disease-free, or overall survival across groups, which included 199 (AC only), 202 (AC with CMF reintroduction), and 194 (CMF only) patients, respectively. Distant disease-free survival was $74 \%$ in the group with CMF only and $70 \%$ (AC only) and 79\% (AC with subsequent CMF) in the others. Survival was $86 \%$ in women with CMF, $90 \%$ in those with $\mathrm{AC}$ only, and $84 \%$ in women receiving
AC with CMF reintroduction ${ }^{1}$. Similarly, Wallgren et al. [20] used data from two International Breast Cancer Study Group (IBCSG) trials (IBCSG VI and VII) collected from 1986 to 1993 on women who received breast conserving surgery to see whether or not it is safe to delay radiation therapy until completion of chemotherapy in women with node-positive disease. Both trials stratified patients by type of surgery. In IBCSG trial VI, which is the most relevant for the purpose of studying the impact of delay to radiation since all patients received some chemotherapy, 1554 pre- or perimenopausal node-positive women were assigned to either three or six courses of CMF with or without subsequent reintroduction. In trial VII, 1266 postmenopausal node-positive patients were allocated to receive either tamoxifen for 5 years, or tamoxifen for 5 years with three early cycles of CMF, both with or without three courses of delayed CMF. The analysis was restricted to women undergoing BCS plus radiation therapy, received after a median delay of 4 and 6.6 months in trial VI, and 2.3 and 3.9 months in trial VII. No differences in the 4-year percent of total failure (25\% versus $30 \%$ in pre/perimenopausal women after 3 versus 6 cycles of CMF, 23\% versus $21 \%$ in postmenopausal women without versus with initial three cycles of CMF), total distant failure (18\% versus $23 \%, 18 \%$ versus $15 \%$, respectively), and overall survival (90\% versus $84 \%, 89 \%$ versus $89 \%$, respectively) were observed. Although the proportion of women receiving chemotherapy differed, both trials reached the same conclusion. The results were similar when the analysis was restricted to women with at least 4 years of follow-up who actually received radiation therapy, but the numbers of cases then became very small, 90 and 107 in women from trial VI with three or six cycles of CMF and 65 and 68 in trial VII without and with CMF. Rates of local recurrences (with or without other sites) were 8 and $7 \%$ in trial VI and 3 and $6 \%$ in trial VII in the early and late RT groups. There were no differences between treatments within any subgroups defined by age, ER or degree of nodal involvement. The authors concluded that delaying radiation therapy after completion of chemotherapy does not adversely influence treatment outcomes.

One study [21] compared patients who received chemotherapy to patients who did not. The effect of delaying radiation on recurrences is then highly confounded by systemic treatment received. No effect of

\footnotetext{
1 Percentages estimated from Figure 2 in [19].
} 
delay on outcomes was observed. It included 471 patients with stage I or II disease treated with wide local excision between 1980 and 1990 at Memorial Hospital in New York. A total of 332 patients were treated with breast-conserving surgery followed by radiation therapy only, with no further systemic treatment. This group had a median follow-up of 77 months and is designated as the RT only group. A second group of 53 patients received all chemotherapy before radiation (the CT first group). The regimens used varied over time. The CAF/CMFVP regimen was used through the late 1980 s for women with four or more positive nodes, whereas CMF (6-8 cycles) was used for patients with three or less positive nodes, including node-negative patients, in the latter part of the 1980s. Until the mid-1980s, sandwich radiotherapy (one to three cycles of CMF before radiation, the rest after radiotherapy for a total of six cycles) was used in 86 node-positive patients. The median follow-up time of these last two groups was 53 and 69 months. No patient in this study received radiation first followed by chemotherapy. Radiation therapy was delivered to the whole breast and dose varied between 45 and $50 \mathrm{~Gy}$, plus a boost of between 14 and $22 \mathrm{~Gy}$. The two groups with chemotherapy had comparable proportions of node-negative cases (23\%), as compared to $86 \%$ of women in the RT only group, and on average, they were 6 years younger. The median interval time before initiation of radiation therapy was 6 weeks in the RT only group, 29 weeks in the CT first group, and 7 weeks in the third group. Although at 5 years a trend was noted in terms of increased local failure in the CT first group, it did not reach significance. The hazard ratio, adjusted for nodal status and $\mathrm{T}$ stage, was $0.69(0.17,2.83)$ for local failure among women receiving chemotherapy (first or split) versus radiation therapy only. The same estimate for distant metastasis was $1.35(0.50,3.65)$.

Two other observational studies [22, 23], both including very small samples, were restricted to patients who all received chemotherapy without tamoxifen. The first one [22] included 105 patients all treated from 1980 to 1990 at the University of Washington Medical Center with chemotherapy and radiation therapy for locoregional breast cancer. Forty-eight patients received their radiation treatment within 6 months, and 57 more than 6 months after their diagnosis. Only $15 \%$ of cases in each group were node-negative. Surgery was conservative or biopsy alone in $60 \%$ of women and $40 \%$ had total mastectomy. Radiation was delivered at doses of 45-50 Gy, usually 5 days a week, at daily doses of 1.8-2 Gy. Boosts of 10-24 Gy were delivered to women felt to be at high risk of residual disease. The majority of patients in the early irradiation group received one or two cycles of chemotherapy prior to radiation. In the delayed radiation group, all patients received chemotherapy within 3 months of their diagnosis. Combination therapy was used and a greater proportion of patients in the delayed irradiation group received a doxorubicin-based regimen.

Prognostic variables were similar in both groups, except for a larger proportion of patients with close or positive margins in the early irradiation group $(69 \%$ versus $38 \%, p<0.02$ ). At 8 years, local control rates were $98 \%$ for the early irradiation group and $76 \%$ for the other one, a highly significant difference $(p=0.004)$, and this remained significant after adjustment for prognostic factors. Disease-free and overall survival rates were 71 and $80 \%$ in the early group, as compared with 48 and $52 \%$ for the delayed one, $p=0.008$ and 0.016. All these trends were observed within each category of surgical treatment, but reached significance only in the group of women with breast conserving surgery. The second study included 84 node-positive patients treated at Rush-Presbyterian-St. Luke's Medical Center between 1977 and 1989 [23]. They had lumpectomy, node dissection, radiation therapy, and chemotherapy. Forty-two patients received radiation therapy before 120 days, and 42 more than 120 days after surgery. In the first group, the majority of patients received CMF $(n=32)$, six received either $\mathrm{AC}$ or $\mathrm{CAF}$, and 4 some other regimen. In the delayed group, 29 patients received CMF, 12 received CAF, and one received L-PAM/5-FU. Radiation therapy was delivered at a median dose of $45 \mathrm{~Gy}$ and 37 patients, 17 in the early group and 20 in the late one, received a boost of 6-30 Gy to the tumor bed. More patients were given supraclavicular treatment in the delayed than the early irradiation group (34/43 versus 26/42). Finally, internal mammary nodes were treated in six patients in each group. The 5-year actuarial local recurrence rate was $2 \%$ in the early group and $14 \%$ in the delayed one, a borderline significant difference $(p=0.05)$. All local recurrences were detected within 30 months of diagnosis. The 5-year actuarial survival rates were 87 and $82 \%$, respectively $(p=0.39)$, whereas diseasefree survival and distant disease-free survival were 85 and $85 \%$ for the early group, compared with 69 and $81 \%$ for the delayed group ( $p=0.04$ and 0.34 , respectively). In multivariate analysis adjusting for prognostic factors, margin of excision was the only factor predictive of local recurrence $(p=0.04)$. The 
surgery-to-radiation interval was also influential but did not reach significance $(p=0.09)$. Using survival as the end point, only number of positive lymph nodes and tumor size were significant predictors of survival ( $p=0.02$ and 0.04 , respectively). The authors concluded that initiation of radiation therapy should be fewer than 120 days after surgery. These two studies are fairly similar with regard to their case mix as well as treatments provided, susceptibility to selection bias, and attempt to control for any prognostic imbalance between groups by the use of multivariate analytical models. Although their conclusions regarding local recurrences are compatible, low statistical power and differences in length of followup could partially explain why delay to radiation was a determinant of survival in one but not in the other.

Finally, five studies [24-28] focussed on populations with different levels of exposure to systemic therapy, and except for one [25], provided data within homogeneous subgroups of treatment or used multivariate analytical models to adjust for differences in exposure to systemic therapy. With one exception [28], all concluded to no impact of delaying radiation on outcomes.

Leonard et al. [24] compared two groups of patients treated at the University of Colorado Health Sciences Center between 1982 and 1992. The first group $(n=105)$ received all or part of chemotherapy before irradiation, on average 5 months after surgery. The second group $(n=157)$ received radiation therapy only, at a median of 1.5 months following definitive surgery. A minority of cases had quadrantectomy or segmental mastectomy. Radiation therapy was delivered to the whole breast at daily doses of 1.8-2.0 Gy for a total of 45-50 Gy. Seventy-six percent of patients received a boost to the tumor bed. Chemotherapy included CMF in a majority of patients $(91 \%)$. Eighty-nine percent of patients in group I received all chemotherapy before irradiation. Tamoxifen was also taken by 67 and $53 \%$ of women in each group. Patients receiving chemotherapy had a significantly greater number of positive lymph-nodes (45\% versus $12 \%$ ) and younger age (43 versus 56 years), but mean tumor size was similar in both groups. The mean follow-up period was 42 months in group I and 55 in group II. The local recurrence rate was $4 \%$ in group I and 5\% in group II, a nonsignificant difference. Moreover, within each group, it was unaffected by the interval between surgery and radiation therapy, analyzed using several nonoverlapping time periods
( $<1$ month, $\geq 1$ and $<3$ months, $\geq 3$ and $<6$ months, $\geq 6$ months; $<4$ versus $\geq 4$ months; $<6$ versus $\geq 6$ months). For each interval, the recurrence rates were similar in groups I and II. The distant recurrence rate was $7 \%$ in group I and $8 \%$ in group II, the 5-year overall survival rate was 91.5 and $84 \%$; for relapsefree survival, it was 85 and $84 \%$. These outcomes were unaffected by interval to radiation therapy. Using Cox regression analysis, neither the surgery-radiotherapy interval nor age, tumor size, margin status, lymph node positivity, or use of tamoxifen was a significant predictor of local recurrence. Meek et al. [25] conducted a study of 310 tumors in 297 patients with stage 0-II breast cancer treated between 1984 and 1989 at University Hospital, Stony Brook, NY. Surgery was described as quadrantectomy in 40 cases and lumpectomy in 270 others. Radiation therapy was delivered to the whole breast to the usual dose of $50 \mathrm{~Gy}$ in 25 fractions, with a boost to the involved region, usually with 9-12 MeV electrons. Seventy-six cases received adjuvant chemotherapy, 43 before radiation, 20 sandwiched around radiation therapy, 12 after radiotherapy, and one concurrently. One hundred and two cases received tamoxifen, 22 with chemotherapy. In the group of 247 patients not receiving chemotherapy or starting chemotherapy after radiation or concurrently, delay to radiation was 2-59 weeks, average 8 weeks. In the 63 cases with chemotherapyinduced delay, the interval was 12-63 weeks, mean of 31 weeks. Cases with delayed treatment had a higher proportion of T2 $(p=0.02)$, high-grade tumors $(p=0.004)$, and more axillary-node positive $(p<0.001)$, stage II disease $(p<0.001)$. Only one patient who received chemotherapy had in-breast relapse and 10 of 11 cases with an in-breast relapse were in the group without a delay to radiation $(p=0.57)$. There also was no association between pattern of chemotherapy and risk of distant metastasis $(p=0.3)$. The 5-year risk of dying with distant metastases was $4.8 \%$ with delayed treatment and $7.3 \%$ with early therapy, a nonsignificant difference $(p=0.53)$. Because of incomplete pathological reporting, lymphatic permeation, and extensive intraductal component could not be assessed. Moreover, no multivariate analysis was performed to adjust for differences in use of systemic treatment and prognostic factors between groups, making the results of this study difficult to interpret. Ampil et al. [26] compared two groups of 26 stage I and 21 stage II patients treated with wide local excision and radiation therapy without or with adjuvant chemotherapy between 1981 and 1995 in 
Louisiana. Among women without systemic treatment (the NAC group), those receiving radiation within 7 weeks were compared to women irradiated later. In the chemotherapy (AC) group, this threshold was fixed at 24 weeks and chemotherapy was usually administered first. The chemotherapeutic agents usually used were a three-drug combination with adriamycin, cyclophosphamide, methotrexate, and 5-fluorouracil. Radiation therapy was administered to a dose of 46-50 Gy over 4.5-5.5 weeks, 5 days a week at daily doses of 1.8$2.0 \mathrm{~Gy}$. The majority of patients who received nodal irradiation were treated in the 1980s. Among women not receiving chemotherapy, those irradiated within 7 weeks of surgery had local and distant relapse rates ( $0 \%$ in both cases) similar to those treated later (18 and $12 \%, p$-values $>0.40$, and 0.70 , respectively). Contrary to the conclusions of studies restricted to patients unexposed to chemotherapy reported above, 4-year crude survival rates were 78 and $29 \%$, a significant difference $(p=0.05)$. In the AC group, none of the differences in local and distant recurrences or survival were significant. Another study included 221 high-risk patients treated with postmastectomy radiation at the University of Pennsylvania between 1977 and 1992 [27]. These patients were considered at high risk because of a pathologic T3 or T4 tumor (14\%), close or positive margins (15\%), positive lymph nodes $(29 \%)$, or multiple risk factors $(39 \%)$. All had either modified radical mastectomy or radical mastectomy. Adjuvant chemotherapy was given to $68 \%$, hormonal therapy to $53 \% ; 36 \%$ received both treatments. Radiation therapy was delivered to the chest wall to doses of 45-50.4 Gy with tangential fields, with daily doses of 1.8-2.0 Gy over 5-6 weeks. A boost dose to the mastectomy scar was delivered to 14 patients $(6 \%)$. The regional lymph nodes were irradiated according to the policy of the attending physician. Chemotherapy consisted of CMF in 113 patients $(75 \%)$, CAF in 73 patients (48\%), and other combination regimens in five patients (3\%). It was administered sequentially in 113 patients $(75 \%)$, concurrently in $12(8 \%)$, and using a sandwich technique in 26 women $(17 \%)$. In women with concurrent chemoradiation, only CF was administered during radiotherapy. Among patients with a delay to radiation of 2 months or less, 31 (38\%) were node-negative and $27(33 \%)$ had four or more positive nodes; these proportions were 10 and $60 \%$ in women having radiotherapy between 2 and 6 months, and 3 and $74 \%$ in those irradiated more than 6 months after surgery (overall $p<0.001$ ). The actuarial rates of locoregional recurrence at 8 years were 13, 4, and
$12 \%$ among patients receiving radiotherapy 2 months or less, 2.1-6 months, and more than 6 months after surgery, a nonsignificant difference $(p=0.51)$. A larger proportion of patients in the early group did not receive chemotherapy and this could attenuate any difference in outcome associated with delay to radiation. However, the same conclusion was reached in a subset analysis of 151 patients who received chemotherapy (AC with or without paclitaxel) where these percentages were 21,2 , and $9 \%$ with increasing interval to radiation $(p=0.16)$. No multivariate analysis to adjust for differences between groups in prognostic factors, especially nodal status, margins, and hormone receptors, as well as use of tamoxifen was performed.

The only study of this group to suggest a detrimental effect of late radiation was done by Slotman et al. [28] and it involved 508 patients with stage I and II invasive breast carcinomas treated between 1980 and 1989 at a single institution in The Netherlands. All underwent conservative breast surgery with wide local excision, nodal dissection, and radiation therapy. The whole breast was treated using opposed tangential fields to a dose of 50 or $50.4 \mathrm{~Gy}$ and a boost dose of $15 \mathrm{~Gy}$, more in cases with close or positive margins. The axilla was irradiated in 104 cases where a single excision was used for removal of the breast tumor and the axilla, and the internal mammary nodes were irradiated in 275 patients with a medial or central tumor and one or more positive nodes. Nodepositive pre-menopausal patients received chemotherapy ( $n=73$, standard regimen of six courses of CMF at 3-4 weeks interval), whereas 92 postmenopausal women, 76 of them node-positive, received tamoxifen. The median follow-up was 68 months. There were no significant differences in age, T-stage, pathological margins, $\mathrm{N}$-stage, radiation dose to the breast, and percent of patients receiving adjuvant systemic therapy between the groups receiving radiation within and after 50 days of surgery. The locoregional recurrence rate was $1.7 \%$ for patients who started radiotherapy within 50 days and $5.6 \%$ for those with a longer interval $(p<0.05)$. In a Cox proportional hazard analysis, tumor stage, pathological margins, as well as the interval between surgery and start of radiotherapy were independent predictors of recurrence and this last variable remained significant in subgroup analysis restricted to patients who received systemic therapy. The distant failure rate and overall survival were unrelated to the interval until radiation as well as margin status. 


\section{Discussion}

This review of time factors related to outcomes of adjuvant radiation therapy included two types of studies, those on sequencing and those on delay to radiation. The majority of these investigations were retrospective case reviews involving heterogeneous study populations with regard to prognostic factors as well as co-treatments. It is, therefore, often difficult to reconcile discordant observations, especially since survival was only recently recognized as a relevant end-point, and, therefore, was not consistently reported. Theoretically, however, there should be consistency between studies on sequencing of chemotherapy and radiotherapy, and those assessing delay among patients who received both, especially when similar regimens of chemotherapy were used in the groups under comparison.

The only published randomized clinical trial on sequencing [14] supports the notion that delaying radiotherapy is potentially detrimental at least for local control and this should be considered higher-level evidence. Among five publications on sequencing [10-14], three were overall negative [11-13], whereas the other two [10,14] suggested that delaying radiation therapy increases the risk of local failure, but could be justified in high-risk patients in whom early CT has the potential to decrease distant recurrences. Although a trend in the same direction on mortality of early CT was observed, it did not reach statistical significance.

Overall, 11 studies [10, 19-28] provided information on delay to radiotherapy among study populations where chemotherapy was also given, and four [10, 22, $23,28]$ came to conclusions consistent to some extent with data on sequencing. The remainder of these investigations were negative. Methodological factors have certainly contributed to these inconsistencies. Experimental studies were not specifically designed to assess the impact of delaying treatment in women having an indication for radiotherapy, subgroup analyses involved few individuals followed over relatively short periods, and heterogeneity of the chemotherapy regimens used, especially the slight advantage of anthracycline-containing therapy over CMF [19] as used in NSABP B-15, might have been a contributing factor. Several of the negative observational studies reported on small numbers of individuals (139 [21], 105 [24], 151 [27]), followed for periods of time between 4 and 8 years. The definition of late irradiation was fairly consistent (usually about 6 months). However, none of them provided results specific to women with chemotherapy while adjusting for differences in prognostic factors and use of tamoxifen, either by restriction or multivariate analysis including all relevant covariates.

Finally, four studies on delay to radiotherapy in populations who did not receive chemotherapy concluded to a lack of influence of the pattern of care on outcomes after adjustment for other prognostic factors [15-18]. Two of them were restricted to node-negative populations $[16,17]$, and in a third [18], 80\% of participants were node-negative. The period of follow-up, 5 years in all three studies, could have been too short to allow detection of a significant difference between categories of treatment in this low-risk population. The fourth study was restricted to T1 and small T2 cases followed up to 10 years and although time to radiation was a significant predictor of locoregional relapse, this association disappeared after adjustment. In this category of studies, late irradiation was variously defined but never exceeded 3 months; this was substantially shorter than the usual delay observed in patients receiving chemotherapy, which was often 6 months or more. In addition, four [24, 26-28] of the studies listed in Table 3 reported results specific to patients not receiving chemotherapy and two also concluded to a lack of influence of delay to radiation $[24,27]$. The group without chemotherapy in Ampil et al. [26] showed significantly better survival with early radiation, but the comparison involved only nine and 17 patients. Finally, no interaction between systemic therapy (defined as either or both chemotherapy and tamoxifen) and interval between surgery and radiotherapy was observed in Slotman et al. [28] in multivariate analysis adjusting for several prognostic factors, suggesting that longer interval to radiation negatively influences local control independently of systemic therapy.

Four of the studies reported here included patients having mastectomy [11, 13, 22, 27], but only three provided results by type of surgery [13, 22, 27]. Overall, this review, therefore, mostly reflects the experience of women undergoing radiation therapy following breast conserving surgery. However, and although indication for radiotherapy following mastectomy reflects poorer prognosis [3], the outcomes of women so treated were not strikingly different from those having breast conserving surgery when delay to radiation was the main exposure variable.

Pathologic margin status and the use of systemic therapy are the most important factors associated with local recurrences among patients treated with breast 
conserving surgery and radiation therapy [29]. Several studies included in this review confirm the importance of margin involvement either as an independent predictor of local control [17, 23, 28], or as a modifier of the influence of delay to radiation on the rate of local recurrences [14]. It is likely that pathological margin involvement prompts early radiotherapy and this could attenuate toward the null the effect of delay on local control in observational studies.

Meta-analytic results [8] suggest that 6 months represents a critical time limit to radiation, but some of the studies reported here have looked at shorter delays and concluded to differences in outcomes associated with waiting time to radiation as short as 50 days. Infinite delay as found in women who did not receive radiotherapy following breast conserving surgery has been shown to significantly compromise the breast recurrence rate [30]. Although more experimental data would be desirable, the usefulness of observational studies should not be underestimated, given the limited feasibility of testing a large diversity of time thresholds for defining late radiation, the ethical issues associated with the conduct of randomized interventions, and the variability of systemic therapies for breast cancer currently available. The risk of selection bias and confounding by indication represents a major threat to the validity of such investigations, and every attempts should be made to minimize or control for differences in both the distribution of major prognostic factors, especially extent of nodal or margin involvement, and use of systemic therapy likely to influence either or both local and distant disease.

A few theories about the natural history of breast cancer [31, 32] have been proposed to account for the small but definitive risk of recurrences and death in early-stage disease. Recent data on treatment of nodenegative tumors $1 \mathrm{~cm}$ or less conclude to some benefit from systemic therapy with chemotherapy and/or tamoxifen even in these cases at very low risk of recurrence [33]. This supports the notion that a small subset of aggressive breast tumors tend to disseminate very early in the course of this disease, although the best way to detect them by a combination of biomarkers remains to be defined. Overall, there is obvious need to explore new modalities of administration of locoregional and systemic therapies ensuring optimal safety.

Both tamoxifen and chemotherapy have the potential to increase local control, as well as distant disease-free and overall survival [29, 34-38]. The literature on delay to radiation provides some evidence that late radiotherapy has the potential to compromise local control, although this appears to be more likely in high-risk patients. The majority of the studies reported here suggested that radiotherapy is quite effective in controlling microscopic disease independently of time to radiation. However, given the methodological weaknesses identified in this critical appraisal of the available evidence, and with the extension of recommendations for systemic adjuvant therapy to cases previously spared this option [39], it seems prudent to endorse current guidelines, although with radiation therapy administered as early as feasible, especially when the risk of local recurrence is high. Concomitant chemoradiation is an alternative option, but poor cosmetic outcomes and high toxicity associated with some regimens have restricted its use [40-43]. The recent shift toward shorter anthracycline or taxane-based chemotherapy permits earlier radiotherapy and may lead to better local and distant control and survival [3]. More data on the optimal integration of surgery, radiotherapy, and chemotherapy are needed.

\section{Acknowledgement}

This research is supported by the Canadian Breast Cancer Research Initiative.

\section{References}

1. National Institutes of Health: Treatment of early-stage breast cancer. NIH Consensus Conference. JAMA 265: 391-395, 1991

2. Abrams JS, Phillips PH, Friedman MA: Meeting highlights: a reappraisal of research results for the local treatment of early stage breast cancer. J Natl Cancer Inst 87: 1837-1845, 1995

3. Recht A, Edge SB, Solin LJ, Robinson DS, Estabrook A, Fine RE, Fleming GF, Formenti S, Hudis C, Kirshner JJ, Krause DA, Kuske RR, Langer AS, Sledge GW, Whelan TJ, Pfister DG: Postmastectomy radiotherapy: clinical practice guidelines of the American Society of Clinical Oncology. J Clin Oncol 19: 1539-1569, 2001

4. Fortin A, Larochelle M, Laverdière J, Lavertu S, Tremblay D: Local failure is responsible for the decrease in survival for patients with breast cancer treated with conservative surgery and postoperative radiotherapy. J Clin Oncol 17: 101-109, 1999

5. Overgaard M, Hansen PS, Overgaard J, Rose C, Andersson M, Bach F, Kjaer M, Gadeberg CC, Mouridsen HT, Jensen $\mathrm{M}-\mathrm{B}$, Zedeler K: Postoperative radiotherapy in high-risk premenopausal women with breast cancer who receive adjuvant chemotherapy. N Engl J Med 337: 949-955, 1997

6. Ragaz J, Jackson SM, Le N, Plenderleith IH, Spinelli JJ, Basco VE, Wilson KS, Knowling MA, Coppin CML, Paradis M, Coldman AJ, Olivotto IA: Adjuvant radiotherapy and chemotherapy in node-positive premenopausal women with breast cancer. N Engl J Med 337: 956-962, 1997 
7. Overgaard M, Jensen MB, Overgaard J: Postoperative radiotherapy in high-risk postmenopausal breast cancer patients given adjuvant tamoxifen: Danish Breast Cancer Cooperative Group DBCCG82c randomised trial. Lancet 353: 1641-1648, 1999

8. Whelan TJ, Julian J, Wright J, Jadad AR, Levine ML: Does locoregional radiation therapy improve survival in breast cancer? A meta-analysis. J Clin Oncol 18: 1220-1229, 2000

9. Early Breast Cancer Trialists' Collaborative Group: Favourable and unfavourable effects on long-term survival of radiotherapy for early breast cancer: an overview of the randomised trials. Lancet 355: 1757-1770, 2000

10. Recht A, Come SE, Gelman RS, Goldstein M, Tishler S, Gore SM, Abner AL, Vicini FA, Silver B, Connolly JL, Schnitt SJ, Coleman CN, Harris JR: Integration of conservative surgery, radiotherapy, and chemotherapy for treatment of early-stage, node-positive breast cancer: sequencing, timing, and outcome. J Clin Oncol 9: 1662-1667, 1991

11. Nguyen TD, Naja A, Chaplain G, Mere P: Influence du délai entre la chirurgie et l'irradiation locorégionale sur l'évolution des cancers du sein $\mathrm{N}+$ non métastatiques. Une étude du Groupe des radiothérapeutes de la Fédération nationale des centres de lutte contre le cancer. Bull Cancer Radiother 80: 229-233, 1993

12. Buchholz TA, Hunt KK, Amosson CM, Tucker SL, Strom EA, McNeese MD, Buzdar AU, Singletary SE, Hortobagyi GN: Sequencing of chemotherapy and radiation in lymphnode negative breast cancer. Cancer J Sci Am 5: 159-164, 1999

13. Buzdar AU, Kau SW, Smith TL, Ames F, Singletary E, Strom E, McNeese M, Hortobagyi GN: The order of administration of chemotherapy and radiation and its effect on the local control of operable breast cancer. Cancer 71: 3680-3684, 1993

14. Recht A, Come SE, Henderson IC, Gelman RS, Silver B, Hayes DF, Shulman LN, Harris JR: The sequencing of chemotherapy and radiation therapy after conservative surgery for early-stage breast cancer. N Engl J Med 334: 1356-1361, 1996

15. Clarke DH, Lê MG, Sarrazin D, Lacombe M-J, Fontaine F, Travagli J-P, Levin-May F, Contesso G, Arriagada R: Analysis of loco-regional relapses in patients with early breast cancers treated by excision and radiotherapy: experience of the Institut Gustave-Roussy. Int J Radiat Oncol Biol Phys 11: 137-145, 1985

16. Nixon AJ, Recht A, Neuberg D, Connolly JL, Schnitt S, Abner A, Harris JR: The relation between the surgery-radiotherapy interval and treatment outcome in patients treated with breastconserving surgery and radiation therapy without systemic therapy. Int J Radiat Oncol Biol Phys 30: 17-21, 1994

17. Vujovic O, Perera F, Dar AR, Stitt L, Yu E, Voruganti SM, Truong PT: Does delay in breast irradiation following conservative breast surgery in node-negative breast cancer patients have an impact on risk of recurrence? Int J Radiat Oncol Biol Phys 40: 868-874, 1998

18. Froud PJ, Mates D, Jackson JSH, Phillips N, Andersen S, Jackson SM, Bryce CJ, Olivotto IA: Effect of time interval between breast-conserving surgery and radiation therapy on ipsilateral breast recurrence. Int J Radiat Oncol Biol Phys 46: 363-372, 2000

19. Fisher B, Brown AM, Dimitrov NV, Poisson R, Redmond C, Margolese R, Bowman D, Wolmark N, Wickerham DL, Kardinal CG, Shibata H, Paterson AHG, Sutherland CM, Robert NJ, Ager PJ, Levy L, Wolter J, Wozniak T, Fisher ER, Deutsch M: Two months of doxorubicin-cyclophosphamide with and without reinduction therapy compared with 6 months of cyclo- phosphamide, methotrexate, and fluorouracil in positive-node breast cancer patients with tamoxifen-nonresponsive tumors: results from the National Surgical Adjuvant Breast and Bowel Project B-15. J Clin Oncol 8: 1483-1496, 1990

20. Wallgren A, Bernier J, Gelber RD, Goldhirsch A, Roncadin M, Joseph D, Castiglione-Gertsch M: Timing of radiotherapy and chemotherapy following breast conserving surgery for patients with node-positive breast cancer. Int J Radiat Oncol Biol Phys 35: 649-659, 1996

21. McCormick B, Norton L, Yao TJ, Yahalom J, Petrek JA: The impact of the sequence of radiation and chemotherapy on local control after breast-conserving surgery. Cancer J Sci Am 2: 39-45, 1996

22. Buchholz TA, Austin-Seymour MM, Moe RE, Ellis GK, Livingston RB, Pelton JG, Griffin TW: Effect of delay in radiation in the combined modality treatment of breast cancer. Int $\mathrm{J}$ Radiat Oncol Biol Phys 26: 23-35, 1993

23. Hartsell WF, Recine DC, Griem KL, Murthy AK: Delaying the initiation of intact breast irradiation for patients with lymph node positive breast cancer increases the risk of local recurrence. Cancer 76: 2497-2503, 1995

24. Leonard CE, Wood ME, Zhen B, Rankin J, Waitz DA, Norton L, Howell K, Sedlacek S: Does administration of chemotherapy before radiotherapy in breast cancer patients treated with conservative surgery negatively impact local control? J Clin Oncol 13: 2906-2915, 1995

25. Meek AG, Park TL, Weiss TA, Bethune WA: Effect of delayed radiation therapy on local control in breast conservation therapy. Radiology 200: 615-619, 1996

26. Ampil FL, Burton GV, Li BDL, Mills GM: Radiotherapy with and without chemotherapy after breast conservation surgery for early stage breast cancer: a review of timing. CME J Gynecol Oncol 20: 254-257, 1999

27. Metz JM, Schultz D, Fox K, Matthews A, Glick J, Solin LJ: Analysis of outcomes for high-risk breast cancer based on interval from surgery to postmastectomy radiation therapy. Cancer J 6: 324-330, 2000

28. Slotman BJ, Meyer OWM, Njo KH, Karim ABMF: Importance of timing of radiotherapy in breast conserving treatment for early stage breast cancer. Radiat Oncol 30: 206-212, 1994

29. Park CC, Mitsumori M, Nixon A, Recht A, Connolly J, Gelman R, Silver B, Hetelekidis S, Abner A, Harris JR, Schnitt SJ: Outcome at 8 years after breast-conserving surgery and radiation therapy for invasive breast cancer: influence of margin status and systemic therapy on local recurrence. J Clin Oncol 18: 1668-1675, 2000

30. Dalberg K, Mattson A, Rutqvist LE, Johansson U, Riddez L, Sandelin K: Breast conserving surgery for invasive breast cancer: risk factors for ipsilateral breast tumor recurrences. Breast Cancer Res Treat 43: 73-86, 1997

31. Fisher B: Laboratory and clinical research in breast cancer - a personal adventure: the David A. Karnofsky Memorial Lecture. Cancer Res 40: 3863-3874, 1980

32. Hellman S: Natural history of small breast cancers. Karnofsky Memorial Lecture. J Clin Oncol 12: 2229-2234, 1994

33. Fisher B, Dignam J, Tan-Chiu E, Anderson S, Fisher ER, Wittliff JL, Wolmark N: Prognosis and treatment of patients with breast tumors of one centimeter or less and negative axillary lymph nodes. J Natl Cancer Inst 93: 112-120, 2001

34. Early Breast Cancer Trialists' Collaborative Group: Polychemotherapy for early breast cancer: an overview of the randomised trials. Lancet 352: 930-942, 1998 
35. Early Breast Cancer Trialists' Collaborative Group: Tamoxifen for early breast cancer: an overview of the randomised trials. Lancet 351: 1451-1467, 1998

36. Freedman G, Fowble B, Hanlon A, Nicolaou N, Fein D, Hoffman J, Sigurdson E, Boraas M, Goldstein L: Patients with early stage invasive cancer with close or positive margins treated with conservative surgery and radiation have an increased risk of breast recurrence that is delayed by adjuvant systemic therapy. Int J Radiat Oncol Biol Phys 44: 1005-1015, 1999

37. Elkhuizen PHM, van Slooten H-J, Clahsen PC, Hermans J, van de Velde CJH, van den Broek LCJM, van de Vijver MJ, Cooperating Investigators: High local recurrence risk after breast-conserving therapy in node-negative premenopausal breast cancer patients is greatly reduced by one course of perioperative chemotherapy: a European Organization for Research and Treatment of Cancer Breast Cancer Study Group. J Clin Oncol 18: 1075-1083, 2000

38. Recht A, Gray NE, Davidson NE, Fowble BL, Solin LJ, Cummings FJ, Falkson G, Falkson HC, Taylor SG, Tormey DC: Locoregional failure 10 years after mastectomy and adjuvant chemotherapy with or without tamoxifen without irradiation: experience of the Eastern Cooperative Oncology Group. J Clin Oncol 17: 1689-1700, 1999
39. Adjuvant therapy for breast cancer. NIH Consens Statement Online 17: 1-23, 2000

40. Markiewicz DA, Schultz DJ, Haas JA, Harris EER, Fox KR, Glick JH, Solin LJ: The effects of sequence and type of chemotherapy and radiation therapy on cosmesis and complications after breast conservation therapy. Int J Radiat Oncol Biol Phys 35: 661-668, 1996

41. Lamb D, Atkinson C, Joseph D, O'Brien P, Ackland S, Bonaventura A, Dady P, Hamilton C, Spry N, Stewart J, Denham J: Simultaneous adjuvant radiotherapy and chemotherapy for stage I and II breast cancer. Aust Radiol 43: 220-226, 1999

42. Dubey A, Recht A, Come SE, Gelman RS, Silver B, Harris JR, Shulman LN: Concurrent CMF and radiation therapy for early stage breast cancer: results of a pilot study. Int J Radiat Oncol Biol Phys 45: 877-884, 1999

43. Recht A, Harris JR, Come SE: Sequencing of irradiation and chemotherapy for early-stage breast cancer. Oncology 8: 19-28, 1994

Address for offprints and correspondence: N. Hébert-Croteau, Institut national de santé publique du Québec, 4835, rue Christophe Colomb, Montréal, Qc H2d 368, Canada; Tel.: (514) 597-0606, Ext. 4419; Fax: (514) 597-1616; E-mail: nicole.hebertcroteau@inspq.qc.cq 